\title{
News from Universities - Nouvelles universitaires
}

\section{Universitätsnachrichten}

Sweden - Suède - Schweden Stockholm : Prof. Dr. med. Hjalmar Holmgren,

Abteilungsvorsteher für experimen-telle Histologie am Histologischen Institut des KarolinskaInstitutes, ist am 21. Februar 1951 im Alter von 45 Jahren an einem Carcinom verstorben.

Switzerland - Suisse - Schweiz

Lausanne: Als Nachfolger des emeritierten Prof. Dr. N. Popoff, wurde Prof. Dr. G. Wínckler, Strasbourg, zum a. o. Professor der Anatomie und Direktor des Anatomischen Institutes ernannt. New Books - Livres Nouveaux - Buchbesprechungen

Kolb W.: Geschichte des Anatomischen Unterrichtes an der Universität zu Basel.

Verlag Benno Schwabe, Basel 1951. 25 Abb., 214 Seiten. sFr. 16.-.

Jeder einstige Schüler der Basler Medizinischen Fakidtät wird gerne diese ,, Geschichte des anatomischen Unterrichtes an der Universität zu Basel" lesen. und Bewegungsapparates für die Beanspruchung der Röhrenknochen 227

Le tractus iliotibial représente la membrure tendue pour la diaphyse fémoralé et réduit par consequent sa soUicitation. Le muscle biarticulaire décharge Tos long sur toute sa longueur, tandis que le muscle monarticulaire ne décharge que la partie de $\Gamma$ os qu'il enjambe. Les muscles n'ont done pas seulement une fonction cinétique mais aussi une fonction statique très importante. L'auteur présente, pour terminer, à $\Gamma$ aide d'un schema simpli-fié du squelette de la jambe, Tinfluence déchargeante de différents principes de construction sur la soUicitation des os longs et des grandes articulations de la jambe.

\section{LITERATUR}

Pauwels, F.: Z. Anat. u. Entwicklungsgesch., 114, 129, 1948. - Id.: Z. Anat. u.

Entwicklungsgesch. 114, 525, 1950. - Id.: Z. Anat. u. Entwicklungsgesch. 115, 327, 1950. Eingegangen 15. November 1948. 